\title{
AGUA DE COCO (COCUS NUCIFERA) COMO DILUYENTE PARA SEMEN FRESCO DE CONEJO EN LA INSEMINACIÓN ARTIFICIAL
}

\author{
COCONUT WATER (COCUS NUCIFERA) AS A DILUENT FOR RABBIT FRESH SEMEN \\ INARTIFICIAL INSEMINATION
}

\author{
Trejo, C.A. ${ }^{1 *}$; Meza, V.V.M. ${ }^{1}$; Antonio, E.C. ${ }^{1}$; Cotera, R.J. ${ }^{1}$ y Antonio-Cisneros, C.M. ${ }^{1}$ \\ 1Universidad del Papaloapan. Loma Bonita. Oaxaca. México. *atrejo@unpa.edu.mx
}

\author{
Palabras clave adicionales \\ Gazapos. Motilidad. Viabilidad.
}

\section{RESUMEN}

Se estudió el efecto de dos diluyentes, agua de coco y Medio Brackett-Oliphant (MBO), en dilución $1: 1(\mathrm{v} / \mathrm{v})$ sobre la motilidad y viabilidad espermática y parámetros reproductivos en conejas inseminadas artificialmente. La motilidad y viabilidad fueron evaluadas antes y después de la dilución, y a los 30, 60, 90, 120, 180 y $240 \mathrm{~min}$ de refrigeración a $4{ }^{\circ} \mathrm{C}$. La motilidad y la viabilidad no mostraron diferencias significativas al hacer la dilución con los diluyentes evaluados ni durante la refrigeración. La fertilidad observada utilizando semen fresco en la inseminación artificial fue 88 $\%$ para el agua de coco y $80 \%$ para el MBO. E número de gazapos obtenidos fue de 8,89 cuando se utilizó el agua de coco y 7,44 con el MBO $(p<0,05)$.

\section{SUMMARY}

The effect of two diluents; coconut water and Brackett-Oliphant Medium (MBO) at 1:1 ( $/ / v)$ on sperm motility, viability and reproductive parameters in rabbits artificially inseminated was studied. The motility and viability were assessed before and after dilution, and at $30,60,90,120,180$ and $240 \mathrm{~min}$ after refrigeration at $4{ }^{\circ} \mathrm{C}$. The motility and viability showed no significant differences neither at the time of dilution nor during refrigerating period. The fertility observed during artificial insemination was $88 \%$ for coconut water and 80 $\%$ for MBO. The number of born rabbits showed statistic differences $(p<0.05)$, it was greater (8.89) when using coconut water than with the MBO (7.44).

Recibido: 1-12-11. Aceptado: 23-4-12.

\author{
AdDitiOnAL KEYWORDS \\ Kit. Motility. Viability.
}

\section{INTRODUCCIÓN}

La inseminación artificial (IA) con semen fresco ha sido utilizada con gran éxito en ovinos (O'Hara et al., 2010), porcinos y camellos (Morton et al., 2011). En una explotación comercial de conejos, la IA se realiza pocas horas después de haber obtenido el semen. En los últimos años se han realizado progresos significativos en las técnicas de conservación (Roca et al., 2000; Nagy et al., 2002; López-Gatius et al., 2005) y en los protocolos de congelación (Mocé et al., 2005) del semen de conejo, sin embargo la fertilidad y prolificidad son inferiores a las que se obtienen con semen fresco (Viudes de Castro y Vicente, 1996). Uno de los factores que determinan el éxito de la IA en fresco es el uso de diluyentes naturales o sintéticos que permiten el fraccionamiento del eyaculado en varias dosis y mantienen la viabilidad de los espermatozoides. Los diluyentes deben ser de bajo costo y fácil elaboración. Por otra parte, el agua de coco ha sido utilizada como diluyente durante la conservación de semen de ovino (Gutiérrez et al., 2006a) y caprino (Nunes, 1993). El uso de diluyente a base de agua de coco en la congelación de semen tiene un efecto benéfico en la movilidad y viabilidad de los espermatozoides en relación con otros diluyentes naturales como es la leche descremada (Gutiérrez et al., 2006b), ade- 
más de bajo costo y facilidad de preparación (Nunes, 1993). Por lo tanto, el objetivo de este trabajo fue evaluar el uso del agua de coco como diluyente durante la inseminación artificial con semen fresco en conejas.

\section{MATERIAL Y MÉTODOS}

Para este estudio se utilizaron 2 conejos machos y 50 hembras de la raza Nueva Zelanda, de 4 meses edad, alimentados con concentrado Purina ${ }^{\circledR}$ y agua ad libitum. Los diluyentes utilizados $(1: 1, \mathrm{v} / \mathrm{v})$ fueron el MBO (medio de Brackett y Oliphant, 1975) y el agua de coco (Cocus nucifera) (Nunes y Fernández, 2001). Los eyaculados fueron obtenidos dos veces por semana siguiendo la técnica propuesta por Hernández et al. (2008) y mantenidos a una temperatura de 37 ${ }^{\circ} \mathrm{C}$ hasta que se realizó la evaluación de la motilidad y viabilidad en masa (tinción vital con eosina-nigrosina). En el experimento 1, se determinó la motilidad y viabilidad antes y después de la dilución, así como durante el tiempo de refrigeración (240 min). Se obtuvieron un total de 40 ( 20 de cada macho) eyaculados a través de vagina artificial. Cada eyaculado fue dividido en dos alícuotas, una fue diluida con agua de coco y otra con $\mathrm{MBO}$ almacenadas a $4{ }^{\circ} \mathrm{C}$ y se evaluaron la motilidad en masa y viabilidad en los siguientes tiempos 30, 60,90, 120, 180 y $240 \mathrm{~min}$. En el experimento 2, se formaron dos grupos de hembras: unas inseminadas con semen fresco diluido en agua de coco y otras con semen diluido en MBO. Las hembras fueron sincronizadas utilizando $40 \mathrm{UI}$ de PMSG (Folligon, Intervet) $48 \mathrm{~h}$ antes de la inseminación. Después de la inseminación se aplicó 0,2 mL de GnRH (Fertagyl, Intervet) (Arrebola y Fernández, 2009). Se determinó el tiempo de gestación así como el número de gazapos por camada. Se realizó un análisis de varianza para la viabilidad y motilidad en masa con el paquete estadístico SigmaStat (Jandel Corporation).

\section{RESULTADOSYDISCUSIÓN}

Los valores medios obtenidos para los eyaculados fueron los siguientes: volumen, 0,61 $\pm 0,09 \mathrm{~mL}$; concentración, 244,2 $\pm 25,9$ x $10^{6}$ espermatozoides/mL; viabilidad, 89,25 $\pm 2,05 \%$ y motilidad individual, $88,87 \pm 3,7$ $\%$. No se observaron diferencias significativas entre los dos machos utilizados (tabla I). Hernández et al. (2008) reportan una motilidad progresiva y viabilidad de semen fresco de conejo de 82,72 y $90,90 \%$, respectivamente. Los resultados obtenidos en este trabajo son superiores a lo reportado por Hernández et al. (2008) para la motilidad y similares en cuanto a la viabilidad.

Experimento 1. No hubo diferencias significativas en la motilidad y viabilidad de los eyaculados diluidos tanto en agua de coco como con MBO (tabla II). Carluccio et al. (2004) reportan una motilidad y viabilidad de 74,2 y $90,5 \%$ respectivamente, cuando la dilución se hace utilizando el medio MIII (MINITUB, Tiefenbach-Germany). Con ambos diluyentes, la motilidad encontrada en este trabajo es superior y la viabilidad es inferior a la reportada por dicho autor $(84,8$;

Tabla I. Motilidad y viabilidad (media \pm DE) de los eyaculados de cada macho. (Motility and viability (mean $\pm S D$ ) of ejaculates for each male).

\begin{tabular}{lcccc}
\hline & Volumen $(\mathrm{mL})$ & Motilidad $(\%)$ & Viabilidad (\%) & Concentración $\left(10^{6}\right)$ \\
\hline Macho 1 $(\mathrm{n}=20)$ & $0,60 \pm 0,07^{\mathrm{a}}$ & $89,05 \pm 2,87^{\mathrm{a}}$ & $89,85 \pm 1,5^{\mathrm{a}}$ & $223,4 \pm 23,4^{\mathrm{a}}$ \\
Macho 2 $(\mathrm{n}=20)$ & $0,61 \pm 0,06^{\mathrm{a}}$ & $87,65 \pm 3,75^{\mathrm{a}}$ & $89,65 \pm 1,6^{\mathrm{a}}$ & $225,0 \pm 20,3^{\mathrm{a}}$ \\
\hline
\end{tabular}

aLetras iguales en la misma columna, indican ausencia de diferencias $(p>0,05)$. 
Tabla II. Motilidad en masa y viabilidad espermática en semen fresco de conejo, diluido con agua de coco o Medio BrackettOliphant. (Motility and sperm viability in rabbits semen after dilution with coconut water or BrackettOliphant Medium).

\begin{tabular}{lcc}
\hline & Motilidad (\%) & Viabilidad (\%) \\
\hline Agua de coco & $86,5 \pm 4,66^{\mathrm{a}}$ & $84,87 \pm 2,53^{\mathrm{a}}$ \\
MBO & $85,25 \pm 1,6^{\mathrm{a}}$ & $83,5 \pm 2,5^{\mathrm{a}}$ \\
\hline
\end{tabular}

aLetras iguales en la misma columna indican ausencia de diferencias $(p>0,05)$.

83,5 vs. $90,5 \%$, respectivamente). Esta diferencia de viabilidad podría deberse a que el diluyente utilizado por Carluccio et al. (2004) era sintético y, por tanto, mucho más complejo que el agua de coco.

Tanto la motilidad como la viabilidad, no mostraron diferencias significativas $(p>$ $0,05)$ durante el tiempo de refrigeración independientemente del diluyente utilizado (tabla III).

Durante la conservación del semen, se reduce la motilidad y se altera la integridad morfológica de los espermatozoides lo que puede ser el resultado de la acumulación de toxinas metabólicas que producen una perioxidación lipídica de las membranas de los espermatozoides (Aitken y Fisher, 1994). Además el transporte y supervivencia de los espermatozoides son afectados en el tracto reproductivo femenino y por ende se reduce la fecundidad. Vasco et al. (2008) reportaron que los espermatozoides congelados-descongelados no sufren estrés oxidativo durante las primeras $6 \mathrm{~h}$ cuando son mantenidos a temperatura ambiente. En este trabajo los eyaculados no fueron congelados sino refrigerados; se podría sugerir que el proceso de peroxidación no esté ocurriendo en tiempos cortos de refrigeración $(4 \mathrm{~h})$ y por lo tanto la motilidad y viabilidad no se vea afectada.

Experimento 2. En las conejas inseminadas con semen diluido en agua de coco se obtuvo una fertilidad del $88 \%$ y cuando se utilizó el MBO, la fertilidad fue del $84 \%$ ( $p>0,05)$. En las hembras inseminadas empleando agua de coco como diluyente, la duración media de la gestación fue de 30,44 días, y en las que se empleó el medio MBO fue de 30,56 días. El número de gazapos por camada en las hembras inseminadas utilizando agua de coco fue de 8,89 , cifra que cuando se utilizó el MBO se redujo a 7,44 $(\mathrm{p}<0,05)$. Esta diferencia podría estar relacionada con la composición química del agua de coco, así Nunes y Fernández (2001) reportaron la existencia de ácido 3-indolacético que aumenta la tasa de fertilidad.

En conclusión, no se han registrado diferencias entre los diluyentes medio Brackett-Oliphant y el agua de coco en cuan-

Tabla III. Motilidad (M) y viabilidad (V) espermática (\%) de semen fresco de conejo diluido con agua de coco o Medio Brackett-Oliphant refrigerado $a 4^{\circ} \mathrm{C}$. (Sperm motility (M) and viability (V) in percent, during storage at $4{ }^{\circ} \mathrm{C}$ of rabbit semen diluted with coconut water or Brackett-Oliphant Medium).

\begin{tabular}{|c|c|c|c|c|c|c|c|c|c|c|c|c|c|c|}
\hline \multirow{3}{*}{ Diluyente } & \multicolumn{14}{|c|}{ Tiempo de refrigeración (minutos) } \\
\hline & \multicolumn{2}{|c|}{0} & \multicolumn{2}{|c|}{30} & \multicolumn{2}{|c|}{60} & \multicolumn{2}{|c|}{90} & \multicolumn{2}{|c|}{120} & \multicolumn{2}{|c|}{180} & \multicolumn{2}{|c|}{240} \\
\hline & M & V & M & V & M & V & M & V & M & V & M & V & M & V \\
\hline Agua de coco & $86,5^{a}$ & $84,9^{a}$ & $85,4^{a}$ & $83,6^{a}$ & $85,3^{\mathrm{a}}$ & $83,5^{\mathrm{a}}$ & $84,9^{a}$ & $83,2^{\mathrm{a}}$ & $84,5^{a}$ & $83,2^{a}$ & $84,2^{\mathrm{a}}$ & $83,0^{a}$ & $84,2^{\mathrm{a}} \varepsilon$ & $82,7^{\circ}$ \\
\hline MBO & $85,3^{a}$ & $83,5^{a}$ & $84,6^{\mathrm{a}}$ & $83,3^{a}$ & $84,1^{a}$ & $83,3^{a}$ & $83,8^{a}$ & $83,2^{\mathrm{a}}$ & $83,7^{a}$ & $82,9^{a}$ & $83,7^{a}$ & $82,6^{a}$ & $83,7^{a}$ & $82,5^{\circ}$ \\
\hline
\end{tabular}

aLetras iguales en la misma columna indican ausencia de diferencia $(p>0,05)$. 


\section{TREJO, MEZA, ANTONIO, COTERA Y ANTONIO-CISNEROS}

to a motilidad y viabilidad durante las $4 \mathrm{~h}$ de refrigeración, pero sí las hubo en cuanto al número de gazapos por camada $(7,44$ y 8,89, respectivamente). El MBO requiere más

\section{BIBLIOGRAFIA}

Aitken, R. and Fisher, H. 1994. Reactive oxygen species generation and human spermatozoa: the balance of benefit and risk. Bioessays, 16: 259-267.

Arrebola, A.D.F. y Fernández, R.L.A. 2009. Consideraciones prácticas acerca de la calidad del semen de conejo aplicado en estudios de toxicología de la fertilidad. REDVET, 10: 1-15.

Carluccio, A.; Robbe, D.; De Amicis, I.; Contri, A. Tosi, V.; Russo, F. and Paoletti, M. 2004. Artificial insemination in rabbits: laboratory and field trial with three difeferent semen extenders. World Rabbit Sci, 12: 65-79.

Brackett, B. and Oliphant, G. 1975. Capactitation of rabbit spermatozoa in vitro. Biol Reprod, 12: 260-273.

Gutiérrez, A.J.; Palacios, M.M.; Jiménez, C.J.A. y Ramírez, G.J.A. 2006a. Agua de coco, Opuntia $\mathrm{sp}$, leche y sus combinaciones para criopreservar semen ovino. Arch Zootec, 55: 97-100.

Gutiérrez, A.J.; Cosme, R.W.; Jiménez, C.J.A. y Ramírez, G.J.A. 2006b. Agua de coco, suero fetal bovino, Aloe vera y sus combinaciones para criopreservar semen ovino. Arch Zootec, 55: 101-104.

Hernández, P.J.E.; Fernández, R.F.; Reyes, C.J.; Cerezo, P.G.; Echegaray, J.L. y Mendoza, B. 2008. Separación de espermatozoides $Y$ de eyaculado de conejo por medio de gradientes de densidad de albumina sérica humana. Rev Salud Anim, 30: 45-49.

López-Gatius, F.; Sances, G.; Sancho, M.; Yániz, J.; Santolaria, P.; Gutiérrez, R.; Núñez, M., Núñez, J. and Soler, C. 2005. Effect of solid storage at $15^{\circ} \mathrm{C}$ on the subsequent motility and fertility of rabbit semen. Theriogenology, 64: 252-260.

Mocé, E.; Lavara, R. and Vicente, J.S. 2005. Influence of the donor male on the fertility of gasto por los diferentes compuestos que se utilizan y más tiempo para su preparación; el agua de coco es más económica y no requiere mucho tiempo de preparación.

frozen-thawed rabbit sperm after artificial insemination of females of different genotypes. Reprod Domest Anim, 40: 516-21.

Morton, K.M.; Billah, M. and Skidmore, J.A. 2011. Effect of green buffer storage on the fertility of fresh camel semen after artificial insemination. Reprod Domest Anim, 46: 554-557.

Nagy, S.Z.; Sinkovics, G.Y. and Kovács, A. 2002. Viability and acrosome integrity of rabbit spermatozoa processed in a gelatin-supplemented extender. Anim Reprod Sci, 70: 283286.

Nunes, J.F. 1993. El agua de coco como diluyente del semen caprino. Revista Cientifica FCVLUZ III, 3: 269-272.

Nunes, J.F. y Fernández, D.R.F. 2001. Biotécnicas de la reproducción caprina y ovina. Editorial Fortaleza. Ceará, Brasil. 105 pp.

O'Hara, L.; Hanrahan, J.P.; Richardson, L.; Donovan, A.; Fair, S.; Evans, A.C. and Lonergan, P. 2010. Effect of storage duration, storage temperature, and diluent on the viability and fertility of fresh ram sperm. Theriogenology, 73: 541-549.

Roca, J.; Martínez, S.; Vázquez, J.M.; Lucas, X.; Parrilla, I. and Martínez, E.A. 2000. Viability and fertility of rabbit spermatozoa diluted in trisbuffer extenders and stored at $15{ }^{\circ} \mathrm{C}$. Anim Reprod Sci, 64: 103-112.

Vasco, D.M.; Hernández, M.M.; Vásquez, J.M.; Martínez, E. y Roca, J. 2008. Sustancias oxígeno reactivas (ROS) en semen congelado-descongelado de porcino. Ciencia y Tecnología, 1: 23-29.

Viudes de Castro, M.P. and Vicente, J.S. 1996. A simple method for freezing rabbit semen with successful results on fertility and prolificity. Anim Reprod Sci, 44: 195-201. 